\title{
Correction to: Estrogen Exerts Neuroprotective Effects in Vascular Dementia Rats by Suppressing Autophagy and Activating the Wnt/ $\beta$-Catenin Signaling Pathway
}

\author{
Yanyan Yang ${ }^{1,2} \circledast$ Lei Zhao $^{3} \cdot \mathrm{Na} \mathrm{Li}^{2} \cdot$ Congwei Dai $^{2} \cdot \mathrm{Nan} \mathrm{Yin}^{3} \cdot$ Zhaoping Chu $^{2} \cdot$ Xiaoyan Duan $^{2} \cdot$ Xiaoli Niu $^{3}$. \\ Ping Yan ${ }^{2} \cdot$ Peiyuan Lv ${ }^{1,3}$
}

Published online: 17 June 2021

(c) Springer Science+Business Media, LLC, part of Springer Nature 2021

\section{Correction to:}

Neurochemical Research (2020) 45:2100-2112

https://doi.org/10.1007/s11064-020-03072-5

In the original version of this article, the complete information of corresponding author Dr. Peiyuan Lv were missed to incorporate.

The complete address of the corresponding author is as below:

Corresponding author, Professor Peiyuan $\mathrm{Lv}^{1,3}$

${ }^{1}$ Department of Neurology, Hebei Medical University, Shijiazhuang 050017, China, and
${ }^{3}$ Department of Neurology, Hebei General Hospital, Shijiazhuang 050051, China.

The authors would like to apologize for the inconvenience caused.

Publisher's Note Springer Nature remains neutral with regard to jurisdictional claims in published maps and institutional affiliations.

The original article can be found online at https://doi.org/10.1007/ s11064-020-03072-5.

Peiyuan Lv

peiyuanlu@163.com

1 Department of Neurology, Hebei Medical University, Shijiazhuang 050017, China

2 Department of Gynecology, Hebei General Hospital, Shijiazhuang 050051, China

3 Department of Neurology, Hebei General Hospital, Shijiazhuang 050051, China 